\title{
Planetary Nebulae and the Cosmic Distance Scale
}

\author{
GEORGE H. JACOBY \\ Kitt Peak National Observatory, NOAO
}

\begin{abstract}
Planetary nebula luminosity functions provide very accurate distances to galaxies as judged by comparisons with other techniques, including Cepheids; systematic effects cannot be significant. The method is empirical and is a consequence of the shallow initial-final mass relationship predicted by stellar evolution and directly measured. From distances to $\sim 30$ galaxies, the PNLF yields $\mathrm{H}_{0}=79$, in excellent agreement with recent Cepheid discoveries in Virgo.
\end{abstract}

Key words: galaxies: distances

\section{Introduction}

Planetary nebula (PN) luminosity functions (PNLF) were suggested as a technique to derive extragalactic distances by Jacoby et al. (1988) and applied to $\sim 30$ galaxies (Jacoby \& Ciardullo 1992). Jacoby (1994) presents the motivation for deriving galaxy distances and intercompares various techniques (e.g., Cepheids, SBF, GCLF, $D_{n}-\sigma, S N$ Ia) with the PNLF distances, demonstrating a high $(\sim 8 \%)$ accuracy and lack of systematic errors. Criteria for judging the quality of distance indicators also are presented, showing that the PNLF satisfies all.

Other new distance methods include surface brightness fluctuations (SBF) and the Type II supernova expanding photosphere method (EPM). The PNLF method offers advantages over these and most other methods.

- The PNLF can be applied to all Hubble types (e.g., LMC, M81, M87)

- Only a single epoch observation is needed

- Reductions are simple; no crowding or lumpy backgrounds to deal with

- Distances are only weakly sensitive to metallicity

- PN are observable from the ground to $\sim 20 \mathrm{Mpc}$

- Internal reddening can be ignored if applied to E/S0 galaxies

- The PN provide kinematic and chemical test probes for follow-up studies

There are two main drawbacks when using the PNLF. It is a secondary distance indicator, and in distant spirals, confusion is possible with compact HII regions unless very high spatial resolution is employed.

\section{Using the PNLF}

The maximum likelihood method yields the distance giving the best match between an adopted PNLF and a target galaxy PNLF. This approach is objective, includes errors on individual PN, is insensitive to outliers (e.g., luminous emissionline sources; see Jacoby \& Ciardullo 1992), and provides objective distance error estimates. It requires that the adopted reference PNLF be appropriate for the target galaxy, and that a zero point be known for the reference PNLF (Jacoby 1994 shows that four Cepheid zero point galaxies exist).

Bottinelli et al. (1991) questioned the appropriateness of the reference PNLF, proposing instead that a power law is a better representation. While true for the brightest $5 \%$ of data, it fits poorly elsewhere; fitting to a luminosity function demands greater generality to prevent torquing the fit by a few outliers. 


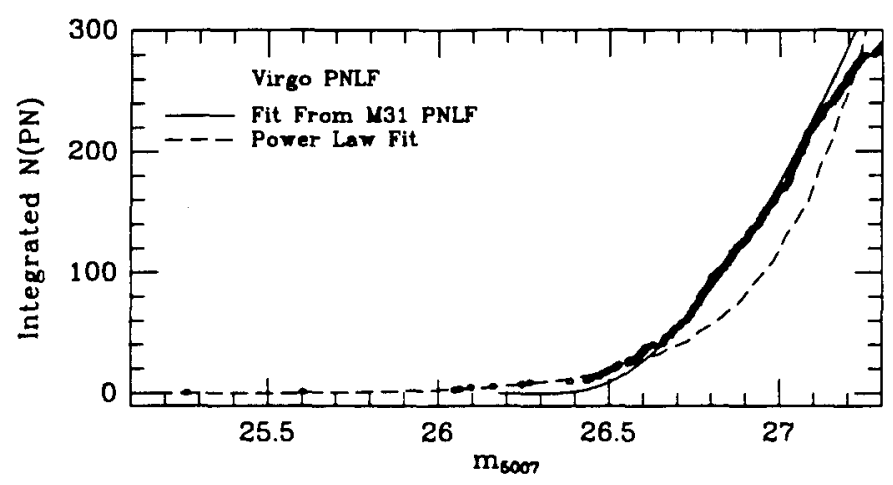

Fig. 1. The integral PNLF for $434 \mathrm{PN}$ in 6 Virgo galaxies. The M31-based fit matches well in the linear part of the curve, deviates slightly for the bright tail, and exceeds the data beyond $m_{5007}=27.1$ where incompleteness begins. Explanations for the bright tail (Jacoby et al. 1990; Jacoby \& Ciardullo 1992) suggest that a revision of the Ciardullo et al. 1989 empirical function is warranted.

Fig. 5 of Bottinelli et al. tries to demonstrate that the PNLF is a power law. Although crowded in their figure, points for each individual galaxy are seen to follow the curve of the Ciardullo et al. (1989) function. Furthermore, a histogram is inadequate for making this comparison. Fig. 1 shows all the Virgo data; this requires presenting an integral PNLF. Overlaid are the proposed power law and the Ciardullo et al. M31-based function. Small deviations from the Ciardullo et al. function exist, but the power law is generally worse. The power law fit can be improved at the faint end, but at the expense of the bright end where the need for the power law originated. Also, detailed studies in the SMC, LMC, M31, and the Galaxy (Méndez et al. 1993) show that a power law is inconsistent with well-studied samples of PN.

\section{Underlying Physics of PNLF Distances}

Jacoby (1989) and Dopita et al. (1991) described the theory behind the stability of the PNLF, the key being the near constancy of stellar core masses (Groenewegen \& De Jong 1993). Higher metallicity decreases the core mass (and luminosity), but increases the nebula's effectiveness to radiate at [OIII] $\lambda 5007$, thus balancing out metallicity differences in populations (Dopita et al. 1991).

From stellar evolution and nebular physics, we find that increasing the luminosity of the PNLF by $0.8 \mathrm{mag}$, the amount required to push the Virgo ellipticals to $22 \mathrm{Mpc}$, demands raising the core masses by $\sim 0.1 \mathrm{M}_{\odot}$ (Jacoby 1989). From Fig. 2, which depicts the evolutionary funneling of the progenitors into white dwarfs, the turn-off masses of many dying stars must increase from $<1.5 \mathrm{M}_{\odot}$ to $>3 \mathrm{M}_{\odot}$. In turn, the population age must be reduced from $>4$ Gyrs to $\sim 100$ Myrs. Thus, the implication of moving M87 from $\sim 15 \mathrm{Mpc}$ to $\sim 22 \mathrm{Mpc}$ is that $\sim 10 \%$ of the light comes from an extremely young population.

With little evidence for $<3$ Gyr populations in Virgo ellipticals (Gonzalez 


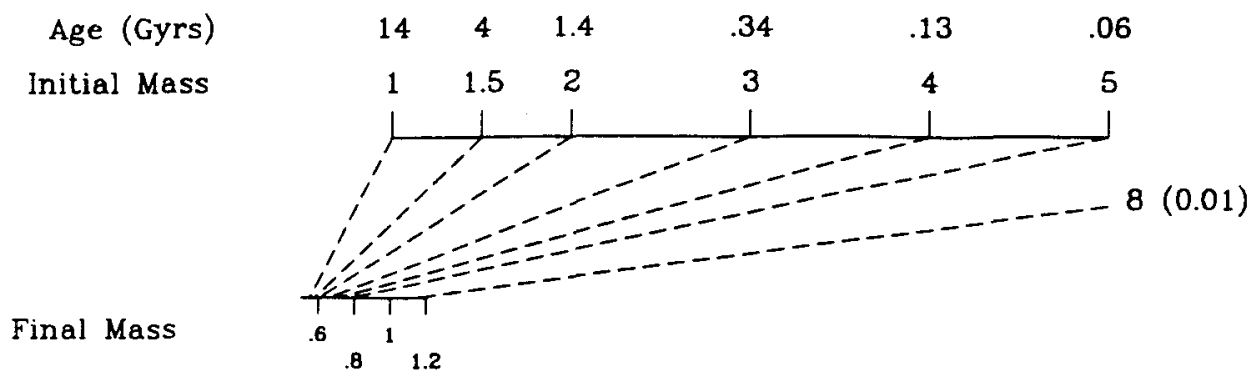

Fig. 2. The initial-final mass relation for main sequence stars based on Weidemann and Koester (1983). Approximate turn-off ages are given.

1993), a long distance to Virgo is unphysical. Also, the excellent distance agreement among Virgo galaxies constrains the age range of the progenitor populations to be $\sim 2 \times$ smaller than Gonzalez finds; alternatively, the final masses could be nearly independent of initial mass, but this conflicts with stellar evolution theory.

\section{Implications for the Cosmic Distance Scale}

From accurate PNLF distances to Virgo, Fornax, and 20 additional galaxies, $\mathrm{H}_{0}=$ $79 \pm 10 \mathrm{~km} / \mathrm{s} / \mathrm{Mpc}$, in excellent agreement with other distance indicators in the same region of space. Type Ia supernovae, assuming that they are all identical, however, yield $\mathrm{H}_{0} \sim 55$, but when a luminosity parameter is used to improve their Hubble relation, $\mathrm{SN}$ Ia yield $\mathrm{H}_{0} \sim 69 \mathrm{~km} / \mathrm{s} / \mathrm{Mpc}$ (Hamuy et al. 1995; Pierce \& Jacoby 1995). Recent Cepheid measurements in Virgo (Pierce et al. 1994) yield a distance of $14.9 \mathrm{Mpc}$, in excellent agreement with the PNLF distance of 15.4 $\mathrm{Mpc}$. Consequently, there is very strong empirical evidence that PNLF distances are correct and $\mathrm{H}_{0} \sim 80$.

\section{References}

Bottinelli, L., Gougenheim, L., Paturel, G., \& Teerikorpi, P. 1991, A\&A, 252, 550.

Ciardullo, R., Jacoby, G.H., Ford, H.C., \& Neill, J.D. 1989, ApJ, 339, 53.

Dopita, M.A., Jacoby, G.H., \& Vassiliadis, E. 1992, ApJ, 389, 27

Gonzalez, J. 1993, PhD Thesis, University of California, Santa Cruz

Groenewegen, M.A.T., \& De Jong, T. 1993, A\&A, 267, 410.

Hamuy, M. et al. 1995, AJ, in press.

Jacoby, G.H. 1989, ApJ, 356, 39.

Jacoby, G.H. 1994, in Science With the VLT, an ESO workshop, ed. J. Walsh, in press.

Jacoby, G.H., \& Ciardullo, R. 1992, in IAU Symposium 155: Planetary Nebulae (Dordrecht: Reidel), ed. R. Weinberger \& A. Acker, p. 503.

Jacoby, G.H., Ciardullo, R., \& Ford, H.C. 1990, ApJ, 356, 332.

Jacoby, G.H., Ford, H.C., \& Ciardullo, R. 1988, in The Extragalactic Distance Scale, A.S.P. Conference Series No. 4, ed. S. van den Bergh \& C.J. Pritchet (Provo: Brigham Young University Press), p.42.

Méndez, R.H., Kudritzki, R.P., Ciardullo, R., \& Jacoby, G.H. 1993, A\&A, 414, 454.

Pierce, M.J. \& Jacoby, G.H. 1995, A J, submitted.

Pierce, M.J. et al. 1994, Nature 371, 385.

Weidemann, V., \& Koester, D. 1983, A\&A, 121, 77. 